\title{
Quantifying channels output similarity with applications to quantum control
}

\author{
Lukasz Pawela ${ }^{1}$ - Zbigniew Puchała ${ }^{1,2}$
}

Received: 25 February 2015 / Accepted: 29 December 2015 / Published online: 12 January 2016

C The Author(s) 2016. This article is published with open access at Springerlink.com

\begin{abstract}
In this work, we aim at quantifying quantum channel output similarity. In order to achieve this, we introduce the notion of quantum channel superfidelity, which gives us an upper bound on the quantum channel fidelity. This quantity is expressed in a clear form using the Kraus representation of a quantum channel. As examples, we show potential applications of this quantity in the quantum control field.
\end{abstract}

Keywords Quantum control · Fidelity · Superfidelity

\section{Introduction}

Recent applications of quantum mechanics are based on processing and transferring information encoded in quantum states. The full description of quantum information processing procedures is given in terms of quantum channels, i.e. completely positive, trace- preserving maps on the set of quantum states.

In many areas of quantum information processing, one needs to quantify the difference between ideal quantum procedure and the procedure which is performed in the laboratory. This is especially true in the situation when one deals with imperfections during the realization of experiments. These imperfections can be countered,

Łukasz Pawela

lpawela@iitis.pl

Zbigniew Puchała

z.puchala@iitis.pl

1 Institute of Theoretical and Applied Informatics, Polish Academy of Sciences, Bałtycka 5, 44-100 Gliwice, Poland

2 Institute of Physics, Jagiellonian University, ulica prof. Stanisława Łojasiewicza 11, 30-348 Kraków, Poland 
in a quantum control setup, using various techniques, such us dynamical decoupling [1-4], sliding mode control [5] and risk sensitive quantum control [6,7]. A different approach is to model the particular setup and optimize control pulses for a specific task in a specific setup [8-11]. In particular, the problem of quantifying the distance between quantum channels was studied in the context of channel distinguishability.

One possible approach to quantifying the distance between two quantum channels is to consider the fidelity between Choi-Jamiołkowski states corresponding to quantum channels [12]. Another approach could involve the diamond norm [13] of quantum channels. We propose an approach which focuses on the outputs of quantum channels.

The main aim of this paper is to provide a succinct expression for the channel output similarity. As a measure of similarity, we will consider the superfidelity function and define channel superfidelity. Then we will show examples of application of our results to various pairs of quantum channels. In the final part of the paper, we will study the impact of Hamiltonian errors on the channel superfidelity. First, we will consider a single qubit at a finite temperature, and next we will move to an extended quantum control example.

\section{Preliminaries}

Henceforth, we will denote the set of linear operators, transforming vectors from a finite-dimensional Hilbert space $\mathcal{X}$ to another finite-dimensional Hilbert space $\mathcal{Y}$ by $\mathcal{L}(\mathcal{X}, \mathcal{Y})$. We put $\mathcal{L}(\mathcal{X})=\mathcal{L}(\mathcal{X}, \mathcal{X})$. By $\mathcal{U}(\mathcal{X})$, we will denote the set of unitary operators on $\mathcal{X}$. Given an operator $A \in \mathcal{L}(\mathcal{X}, \mathcal{Y})$, we denote by $\|A\|_{p}$ its Schatten p-norm. By $\bar{A}$, we will denote the element-wise complex conjugation of $A$.

\subsection{Quantum states and channels}

First, we introduce two basic notions: density operators and superoperatros:

Definition 1 We call an operator $\rho \in \mathcal{L}(\mathcal{X})$ a density operator iff $\rho \geq 0$ and $\operatorname{Tr} \rho=1$. We denote the set of all density operators on $\mathcal{X}$ by $\mathcal{D}(\mathcal{X})$.

From this follows that $\rho$ is in the form $\rho=\sum_{j} \lambda_{j}\left|\lambda_{j}\right\rangle\left\langle\lambda_{j}\right|$, where $\lambda_{j}$ and $\left|\lambda_{j}\right\rangle$ denote the $j$ th eigenvalue and eigenvector of $\rho$, respectively.

Definition 2 A superoperator is a linear mapping acting on linear operators $\mathcal{L}(\mathcal{X})$ on a finite-dimensional Hilbert space $\mathcal{X}$ and transforming them into operators on another finite-dimensional Hilbert space $\mathcal{Y}$. Thus

$$
\Phi: \mathcal{L}(\mathcal{X}) \rightarrow \mathcal{L}(\mathcal{Y})
$$

Now we define the tensor product of superoperators

Definition 3 Given superoperators

$$
\Phi_{1}: \mathcal{L}\left(\mathcal{X}_{1}\right) \rightarrow \mathcal{L}\left(\mathcal{Y}_{1}\right), \Phi_{2}: \mathcal{L}\left(\mathcal{X}_{2}\right) \rightarrow \mathcal{L}\left(\mathcal{Y}_{2}\right)
$$


we define the product superoperator

$$
\Phi_{1} \otimes \Phi_{2}: \mathcal{L}\left(\mathcal{X}_{1} \otimes \mathcal{X}_{2}\right) \rightarrow \mathcal{L}\left(\mathcal{Y}_{1} \otimes \mathcal{Y}_{2}\right)
$$

to be the unique linear mapping that satisfies the equation

$$
\left(\Phi_{1} \otimes \Phi_{2}\right)\left(A_{1} \otimes A_{2}\right)=\Phi_{1}\left(A_{1}\right) \otimes \Phi_{2}\left(A_{2}\right),
$$

for all operators $A_{1} \in \mathcal{L}\left(\mathcal{X}_{1}\right), A_{2} \in \mathcal{L}\left(\mathcal{X}_{2}\right)$.

In the most general case, the evolution of a quantum system can be described using the notion of a quantum channel [14-16].

Definition 4 A quantum channel is a superoperator $\Phi$ that satisfies the following restrictions:

1. $\Phi$ is trace preserving, i.e. $\forall A \in \mathcal{L}(\mathcal{X}) \quad \operatorname{Tr}(\Phi(A))=\operatorname{Tr}(A)$,

2. $\Phi$ is completely positive, that is for every finite-dimensional Hilbert space $\mathcal{Z}$ the product of $\Phi$ and identity mapping on $\mathcal{L}(\mathcal{Z})$ is a non-negativity-preserving operation, i.e.

$$
\forall \mathcal{Z} \forall A \in \mathcal{L}(\mathcal{X} \otimes \mathcal{Z}) \quad A \geq 0 \Rightarrow \Phi \otimes \mathbb{1}_{\mathcal{L}(\mathcal{Z})}(A) \geq 0
$$

Many different representations of quantum channels can be chosen, depending on the application. In this paper, we will use only the Kraus representation.

Definition 5 The Kraus representation of a completely positive superoperator (Def. 4(2)) is given by a set of operators $K_{i} \in \mathcal{L}(\mathcal{X}, \mathcal{Y})$. The action of the superoperator $\Phi$ is given by:

$$
\Phi(\rho)=\sum_{i} K_{i} \rho K_{i}^{\dagger},
$$

This form ensures that the superoperator is completely positive. For it to be also trace preserving, we need to impose the following constraint on the Kraus operators

$$
\sum_{i} K_{i}^{\dagger} K_{i}=\mathbb{1}_{\mathcal{X}},
$$

where $\mathbb{1}_{\mathcal{X}}$ denotes the identity operator acting on the Hilbert space $\mathcal{X}$.

\subsection{Superfidelity}

In this section, we introduce the superfidelity, along with its properties

Definition 6 Superfidelity of two density operators $\rho, \sigma \in \mathcal{D}(\mathcal{X})$ is given by

$$
G(\rho, \sigma)=\operatorname{Tr}(\rho \sigma)+\sqrt{1-\operatorname{Tr} \rho^{2}} \sqrt{1-\operatorname{Tr} \sigma^{2}}
$$


The superfidelity is an upper bound for the fidelity function $[14,17]$.

Properties of the superfidelity [17] $\left(\rho_{1}, \rho_{2}, \rho_{3}, \rho_{4} \in \mathcal{D}(\mathcal{X})\right)$ :

1. Bounds: $0 \leq G\left(\rho_{1}, \rho_{2}\right) \leq 1$.

2. Symmetry: $G\left(\rho_{1}, \rho_{2}\right)=G\left(\rho_{2}, \rho_{1}\right)$.

3. Unitary invariance: $G\left(\rho_{1}, \rho_{2}\right)=G\left(U \rho_{1} U^{\dagger}, U \rho_{2} U^{\dagger}\right)$, where $U \in \mathcal{U}(\mathcal{X})$.

4. Joint concavity [18]:

$$
G\left(p \rho_{1}+(1-p) \rho_{2}, p \rho_{3}+(1-p) \rho_{4}\right) \leq p G\left(\rho_{1}, \rho_{3}\right)+(1-p) G\left(\rho_{2}, \rho_{4}\right)
$$

for $p \in[0,1]$.

5. Supermultiplicavity:

$$
G\left(\rho_{1} \otimes \rho_{2}, \rho_{3} \otimes \rho_{4}\right) \geq G\left(\rho_{1}, \rho_{3}\right) G\left(\rho_{2}, \rho_{4}\right) .
$$

6. Bound for trace distance [19]

$$
\frac{1}{2}\left\|\rho_{1}-\rho_{2}\right\|_{1} \geq 1-G\left(\rho_{1}, \rho_{2}\right) .
$$

\subsection{Supporting definitions}

In this section, we define additional operations used in our proof. We begin with the partial trace

Definition 7 For all operators $A, B$ the partial trace is a linear mapping defined as:

$$
\operatorname{Tr} \mathcal{Y} A \otimes B=A \operatorname{Tr} B
$$

The extension to operators not in the tensor product form follows from linearity.

We will also need the notion of conjugate superoperator

Definition 8 Given a quantum channel $\Phi: \mathcal{L}(\mathcal{X}) \rightarrow \mathcal{L}(\mathcal{Y})$, for every operator $A \in$ $\mathcal{L}(\mathcal{X}), B \in \mathcal{L}(\mathcal{Y})$, we define the conjugate superoperator $\Phi^{\dagger}: \mathcal{L}(\mathcal{Y}) \rightarrow \mathcal{L}(\mathcal{X})$ as the mapping satisfying

$$
\operatorname{Tr}(\Phi(A) B)=\operatorname{Tr}\left(A \Phi^{\dagger}(B)\right) .
$$

Note that the conjugate to completely positive superoperator is completely positive, but is not necessarily trace preserving

Next, we will define a reshaping operation, which preservers the lexicographical order and its inverse.

Definition 9 We define the linear mapping

$$
\text { res }: \mathcal{L}(\mathcal{X}, \mathcal{Y}) \rightarrow \mathcal{Y} \otimes \mathcal{X}
$$

for dyadic operators as

$$
\operatorname{res}(|\psi\rangle\langle\phi|)=|\psi\rangle \overline{|\phi\rangle},
$$

for $|\psi\rangle \in \mathcal{Y}$ and $|\phi\rangle \in \mathcal{X}$ and uniquely extended by linearity. 
We introduce the inverse of the res(.)

Definition 10 We define the linear mapping

$$
\text { unres }: \mathcal{Y} \otimes \mathcal{X} \rightarrow \mathcal{L}(\mathcal{X}, \mathcal{Y})
$$

such that

$$
\forall X \in \mathcal{L}(\mathcal{X}, \mathcal{Y}) \quad \text { unres }(\operatorname{res}(X))=X .
$$

Remark 1 For every choice of Hilbert spaces $\mathcal{X}_{1}, \mathcal{X}_{2}, \mathcal{Y}_{1}$ and $\mathcal{Y}_{2}$ and every choice of operators $A \in \mathcal{L}\left(\mathcal{X}_{1}, \mathcal{Y}_{1}\right), B \in \mathcal{L}\left(\mathcal{X}_{2}, \mathcal{Y}_{2}\right)$ and $X \in \mathcal{L}\left(\mathcal{X}_{2}, \mathcal{X}_{1}\right)$, it holds that:

$$
(A \otimes B) \operatorname{res}(X)=\operatorname{res}\left(A X B^{\mathrm{T}}\right)
$$

Remark 2 For any choice of Hilbert spaces $\mathcal{X}$ and $\mathcal{Y}$ and any choice of $|\zeta\rangle \in \mathcal{X} \otimes \mathcal{Y}$ and $A \in \mathcal{L}(\mathcal{Y}, \mathcal{X})$ such that $|\zeta\rangle=\operatorname{res}(A)$ it holds that

$$
\operatorname{Tr} \mathcal{Y}|\zeta\rangle\langle\zeta|=\operatorname{Tr} \mathcal{Y}\left(\operatorname{res}(A) \operatorname{res}(A)^{\dagger}\right)=A A^{\dagger}
$$

Next, we introduce the purification of quantum states:

Definition 11 Given Hilbert spaces $\mathcal{X}$ and $\mathcal{Y}$, we will call $|\zeta\rangle \in \mathcal{X} \otimes \mathcal{Y}$ a purification of $\rho \in \mathcal{D}(\mathcal{X})$ if

$$
\operatorname{Tr}_{\mathcal{Y}}|\zeta\rangle\langle\zeta|=\rho
$$

Theorem 1 For every choice of Hilbert spaces $\mathcal{X}$ and $\mathcal{Y}$ and let $|\phi\rangle,|\psi\rangle \in \mathcal{X} \otimes \mathcal{Y}$ satisfy

$$
\operatorname{Tr}_{\mathcal{Y}}(|\phi\rangle\langle\phi|)=\operatorname{Tr}_{\mathcal{Y}}(|\psi\rangle\langle\psi|)
$$

Then there exists a unitary operator $U \in \mathcal{U}(\mathcal{X})$ such that $|\psi\rangle=\left(\mathbb{1}_{\mathcal{X}} \otimes U\right)|\phi\rangle$

From Definition 11, Theorem 1 and Remark 2 we get that given a state $\rho \in \mathcal{D}(\mathcal{X})$ its purification $|\zeta\rangle \in \mathcal{X} \otimes \mathcal{X}$ is given by:

$$
|\zeta\rangle=\left(\mathbb{1}_{\mathcal{X}} \otimes U\right) \operatorname{res}(\sqrt{\rho}) .
$$

Verification of this equation is straightforward. First, we note that we may omit the term $\left(\mathbb{1}_{\mathcal{X}} \otimes U\right)$. Next we apply Remark 2 which allows us to show that for this choice of $|\zeta\rangle$ we get:

$$
\operatorname{Tr} \mathcal{Y}|\zeta\rangle\langle\zeta|=\sqrt{\rho}(\sqrt{\rho})^{\dagger}=\rho
$$

\subsection{Quantum channel fidelity}

First, we introduce the fidelity and channel fidelity [12]

Definition 12 Given two density operators $\rho, \sigma$ we define the fidelity between $\rho$ and $\sigma$ as:

$$
F(\rho, \sigma)=\|\sqrt{\rho} \sqrt{\sigma}\|_{1}^{2}=(\operatorname{Tr} \sqrt{\sqrt{\rho} \sigma \sqrt{\rho}})^{2}
$$


Definition 13 Quantum channel fidelity of a channel $\Phi: \mathcal{L}(\mathcal{X}) \rightarrow \mathcal{L}(\mathcal{X})$ for some $\sigma$ is defined as:

$$
F_{\text {ch }}(\Phi ; \sigma)=\inf F\left(\xi,\left(\Phi \otimes \mathbb{1}_{\mathcal{L}(\mathcal{Z})}\right)(\xi)\right)
$$

where the infimum is over all Hilbert spaces $\mathcal{Z}$ and all $\xi \in \mathcal{D}(\mathcal{X} \otimes \mathcal{Z})$ such that $\operatorname{Tr}_{\mathcal{Z}} \xi=\sigma$

It can be shown [20] that this infimum is independent of $\xi$ and is given by

$$
F_{\mathrm{ch}}(\Phi ; \sigma)=\sum_{i}\left|\operatorname{Tr}\left(\sigma K_{i}\right)\right|^{2}
$$

where $K_{i}$ form the Kraus representation of $\Phi$.

\section{Our results}

In this section, we present our main theorem and its proof. In the second subsection, we present a quantum circuit that allows one to measure the quantum channel superfidelity without performing full state tomography.

\subsection{Theorem and proof}

Definition 14 Consider two quantum channels $\Phi, \Psi: \mathcal{L}(\mathcal{X}) \rightarrow \mathcal{L}(\mathcal{X})$ and a density operator $\sigma \in \mathcal{D}(\mathcal{X})$. We define the quantum channel superfidelity to be:

$$
G_{\mathrm{ch}}(\Phi, \Psi ; \sigma)=\inf G\left(\left(\Phi \otimes \mathbb{1}_{\mathcal{L}(\mathcal{Z})}\right)(\xi),\left(\Psi \otimes \mathbb{1}_{\mathcal{L}(\mathcal{Z})}\right)(\xi)\right)
$$

where the infimum is over all Hilbert spaces $\mathcal{Z}$ and over all purifications $\xi=|\zeta\rangle\langle\zeta| \in$ $\mathcal{D}(\mathcal{X} \otimes \mathcal{Z})$ of $\sigma$.

The channel superfidelity $G_{\mathrm{ch}}(\Phi, \Psi ; \sigma)$ places a lower bound on the output superfidelity of two quantum channels in the case of the same input states. Henceforth, where unambiguous, we will write the channel superfidelity as $G_{\mathrm{ch}}$.

Theorem 2 Given quantum channels $\Phi, \Psi: \mathcal{L}(\mathcal{X}) \rightarrow \mathcal{L}(\mathcal{X})$ with Kraus forms given by the sets $\left\{K_{i}\right\}_{i}$ and $\left\{L_{j}\right\}_{j}$, respectively, the quantum channel superfidelity is given by:

$$
\begin{aligned}
G_{\mathrm{ch}}= & \sum_{i, j}\left|\operatorname{Tr} \sigma K_{i}^{\dagger} L_{j}\right|^{2}+\sqrt{1-\sum_{i, j}\left|\operatorname{Tr} \sigma K_{i}^{\dagger} K_{j}\right|^{2}} \\
& \times \sqrt{1-\sum_{i, j}\left|\operatorname{Tr} \sigma L_{i}^{\dagger} L_{j}\right|^{2}} .
\end{aligned}
$$


Proof As we limit ourselves only to pure states $\xi$, in order to calculate the superfidelity, we need to compute the following quantities:

$$
\begin{aligned}
\operatorname{Tr}\left(\Phi \otimes \mathbb{1}_{\mathcal{L}(\mathcal{Z})}\right)(|\zeta\rangle\langle\zeta|)\left(\Psi \otimes \mathbb{1}_{\mathcal{L}(\mathcal{Z})}\right)(|\zeta\rangle\langle\zeta|), & \\
& \operatorname{Tr}\left[\left(\Phi \otimes \mathbb{1}_{\mathcal{L}(\mathcal{Z})}\right)(|\zeta\rangle\langle\zeta|)\right]^{2}, \\
& \operatorname{Tr}\left[\left(\Psi \otimes \mathbb{1}_{\mathcal{L}(\mathcal{Z})}\right)(|\zeta\rangle\langle\zeta|)\right]^{2}
\end{aligned}
$$

As the general idea is shared between all of these quantities, we will show here the calculation for the first one. We get

$$
\begin{array}{r}
\operatorname{Tr}\left(\Phi \otimes \mathbb{1}_{\mathcal{L}(\mathcal{Z})}\right)(|\zeta\rangle\langle\zeta|)\left(\Psi \otimes \mathbb{1}_{\mathcal{L}(\mathcal{Z})}\right)(|\zeta\rangle\langle\zeta|)= \\
\operatorname{Tr}|\zeta\rangle\langle\zeta|\left(\Phi^{\dagger} \circ \Psi \otimes \mathbb{1}_{\mathcal{L}(\mathcal{Z})}\right)(|\zeta\rangle\langle\zeta|)= \\
\left\langle\zeta\left|\left(\Phi^{\dagger} \circ \Psi \otimes \mathbb{1}_{\mathcal{L}(\mathcal{Z})}\right)(|\zeta\rangle\langle\zeta|)\right| \zeta\right\rangle,
\end{array}
$$

where the first equality follows from the definition of the conjugate superoperator. The Kraus form of the superoperator $\Phi^{\dagger} \circ \Psi$ is given by the set $\left\{K_{i}^{\dagger} L_{j}\right\}_{i, j}$. Now, we may write $|\zeta\rangle$ as

$$
|\zeta\rangle=\operatorname{res}(\sqrt{\sigma} U)
$$

for some $U \in \mathcal{L}(\mathcal{Z}, \mathcal{X})$ such that $U U^{\dagger}$ is a projector on the image of $\sigma$. We obtain:

$$
\begin{array}{r}
\left\langle\zeta\left|\left(\Phi^{\dagger} \circ \Psi \otimes \mathbb{1}_{\mathcal{L}(\mathcal{Z})}\right)(|\zeta\rangle\langle\zeta|)\right| \zeta\right\rangle=\sum_{i, j}\left|\left\langle\zeta\left|\left(K_{i}^{\dagger} L_{j}\right) \otimes \mathbb{1}_{\mathcal{Z}}\right| \zeta\right\rangle\right|^{2}= \\
\sum_{i, j}\left|\operatorname{res}(\sqrt{\sigma} U)^{\dagger}\left(\left(K_{i}^{\dagger} L_{j}\right) \otimes \mathbb{1}_{\mathcal{Z}}\right) \operatorname{res}(\sqrt{\sigma} U)\right|^{2}= \\
\sum_{i, j}\left|\operatorname{Tr} U^{\dagger} \sqrt{\sigma} K_{i}^{\dagger} L_{j} \sqrt{\sigma} U\right|^{2}=\sum_{i, j}\left|\operatorname{Tr} \sigma K_{i}^{\dagger} L_{j}\right|^{2} .
\end{array}
$$

This quantity is independent of the particular purification of $\sigma$. Following the same path for the other two quantities shown in Eq. (29), we recover the expression for the channel superfidelity from Eq. (28).

Since the superfidelity is an upper bound for the fidelity function, we obtain, the following inequality:

$$
G_{\mathrm{ch}}(\Phi, \Psi ; \sigma) \geq F(\Phi, \Psi ; \sigma),
$$

where $F(\Phi, \Psi ; \sigma)=\inf _{\mathcal{Z}, \xi} F\left(\left(\Phi \otimes \mathbb{1}_{\mathcal{L}(\mathcal{Z})}\right)(\xi),\left(\Psi \otimes \mathbb{1}_{\mathcal{L}(\mathcal{Z})}\right)(\xi)\right)$ and the infimum is over all Hilbert spaces $\mathcal{Z}$ and all $\xi \in \mathcal{D}(\mathcal{X} \otimes \mathcal{Z})$ such that $\operatorname{Tr}_{\mathcal{Z}} \xi=\sigma$.

The following simple corollaries are easily derived from Theorem 2 .

Corollary 1 Given a quantum channel $\Phi$, the superfidelity between its input and output reduces to the channel fidelity of $\Phi$ :

$$
G_{\mathrm{ch}}(\Phi, \mathbb{1} ; \sigma)=F_{\mathrm{ch}}(\Phi ; \sigma)
$$


Proof Assume $\Phi$ has the Kraus form $\left\{K_{i}\right\}_{i}$. Substituting the identity for $L_{j}$ in Eq. (27), we recover Eq. (25) which completes the proof.

Corollary 2 If $\Phi$ is a unitary channel i. e. $\Phi(\rho)=U \rho U^{\dagger}$ for any $U \in \mathcal{U}(\mathcal{X})$ and $\Psi^{\prime}: \rho \mapsto U^{\dagger} \Psi(\rho) U$, where $\Psi$ is an arbitrary quantum channel then

$$
G_{\mathrm{ch}}(\Phi, \Psi ; \sigma)=F_{\mathrm{ch}}\left(\Psi^{\prime} ; \sigma\right)
$$

Proof If $\Phi$ is a unitary channel, then the second term in Eq. (28) vanishes. Let us assume that $\Psi$ has a Kraus form $\left\{L_{j}\right\}_{j}$. We get $G_{\mathrm{ch}}=\sum_{j}\left|\operatorname{Tr} \sigma U^{\dagger} L_{j}\right|^{2}$.

The Kraus form of the channel $\Psi^{\prime}$ is given by the set $\left\{U^{\dagger} L_{j}: K_{j} \in \mathcal{L}(\mathcal{X})\right\}$. Using this in Eq. (25), we get $F_{\mathrm{ch}}\left(\Psi^{\prime} ; \sigma\right)=\sum_{j}\left|\operatorname{Tr} \sigma U^{\dagger} L_{j}\right|^{2}$. This completes the proof.

Corollary 3 If $\sigma \in \mathcal{D}(\mathcal{X})$ is a pure state, i.e. $\sigma=|\psi\rangle\langle\psi|$, then

$$
G_{\mathrm{ch}}(\Phi, \Psi ; \sigma)=G(\Phi(\sigma), \Psi(\sigma))
$$

Proof Let us only focus on the first terms in Eqs. (8) and (28). We will denote these terms $T$ and $T_{\mathrm{ch}}$, respectively. Let us assume that channels $\Phi$ and $\Psi$ have Kraus forms $\left\{K_{i}\right\}_{i}$ and $\left\{L_{j}\right\}_{j}$, respectively. We get:

$$
\begin{aligned}
T & =\operatorname{Tr} \sum_{i j} K_{i}|\psi\rangle\left\langle\psi\left|K_{i}^{\dagger} L_{j}\right| \psi\right\rangle\langle\psi| L_{j}^{\dagger} \\
& =\sum_{i j}\left\langle\psi\left|K_{i}^{\dagger} L_{j}\right| \psi\right\rangle\left\langle\psi\left|L_{j}^{\dagger} K_{i}\right| \psi\right\rangle=\sum_{i j}\left|\left\langle\psi\left|K_{i}^{\dagger} L_{j}\right| \psi\right\rangle\right|^{2}=T_{\mathrm{ch}} .
\end{aligned}
$$

Performing similar calculations for other terms, we recover Eq. (36)

\subsection{Quantum circuit for measuring channel superfidelity}

Using the quantum circuit shown in Fig. 1, we can measure the quantum channel superfidelity in an experimental setup. This setup allows us to estimate the $\operatorname{Tr}\left(\Phi \otimes \mathbb{1}_{\mathcal{L}(\mathcal{Z})}\right)(|\zeta\rangle\langle\zeta|)\left(\Psi \otimes \mathbb{1}_{\mathcal{L}(\mathcal{Z})}\right)(|\zeta\rangle\langle\zeta|)=2 p_{0}-1$, where $p_{0}$ is the probability of finding the top qubit in the state $|0\rangle$. Modifying the circuit appropriately, we can measure all the quantities shown in Eq. (29).

Note that this approach is far simpler, compared to estimating the channel fidelity which would require us to perform full state tomography. Furthermore, analytical calculations involving fidelity get cumbersome quickly, as it requires calculating expressions of the form $\|\sqrt{\Phi(\sigma)} \sqrt{\Psi(\sigma)}\|_{1}$.

\section{Simple examples}

In this section, we provide a number of examples of the application of Theorem 2. 


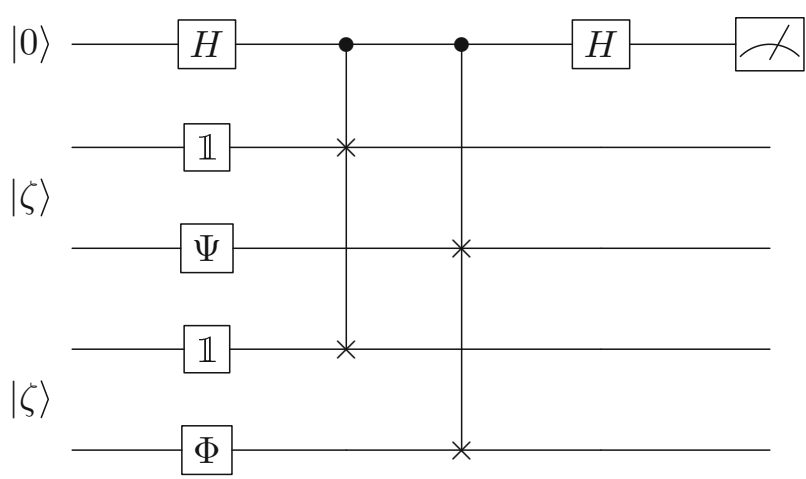

Fig. 1 Quantum circuit for measuring $\operatorname{Tr}\left(\Phi \otimes \mathbb{1}_{\mathcal{L}(\mathcal{Z})}\right)(|\zeta\rangle\langle\zeta|)\left(\Psi \otimes \mathbb{1}_{\mathcal{L}(\mathcal{Z})}\right)(|\zeta\rangle\langle\zeta|)=2 p_{0}-1$, where $p_{0}$ is the probability of finding the top qubit in state $|0\rangle$ [21]. This allows direct estimation of the channel superfidelity

\subsection{Erasure channel}

Definition 15 Given a quantum state $\xi \in \mathcal{D}(\mathcal{X})$, the erasure channel is given by:

$$
\Phi(A)=\xi,
$$

for any $A$ in $\mathcal{L}(\mathcal{X})$. The Kraus form of this channel is given by the set $\left\{K_{i j}: K_{i j}=\right.$ $\left.\sqrt{\lambda_{i}}\left|\lambda_{i}\right\rangle\langle j|\right\}_{i j}, \lambda_{i}$ and $\left|\lambda_{i}\right\rangle$ denote the $i$ th eigenvalue and the corresponding eigenvector of $\xi$.

Let us consider the superfidelity between the erasure channel $\Phi$ and a unitary channel $\Psi: \sigma \mapsto U \sigma U^{\dagger}$ for some $U \in \mathcal{U}(\mathcal{X})$. We note that the second term in Eq. (28) vanishes. What remains is:

$$
\begin{aligned}
G_{\mathrm{ch}} & =\sum_{i j} \lambda_{i}\left|\left\langle j\left|\sigma U^{\dagger}\right| \lambda_{i}\right\rangle\right|^{2}=\sum_{j}\left\langle j\left|\sigma U^{\dagger}\left(\sum_{i} \lambda_{i}\left|\lambda_{i}\right\rangle\left\langle\lambda_{i}\right|\right) U \sigma\right| j\right\rangle \\
& =\operatorname{Tr} \sigma^{2} \Psi^{\dagger}(\xi) \leq \sum_{i} \lambda_{i}^{\downarrow} \mu_{i}^{\downarrow},
\end{aligned}
$$

where $\mu_{i}^{\downarrow}$ and $\lambda_{i}^{\downarrow}$ denote the eigenvalues of $\sigma$ and $\xi$, respectively, sorted in a descending order. The last inequality follows from von Neumann's trace inequality [22].

\subsection{Sensitivity to channel error}

Consider a quantum channel $\Phi$ with the Kraus form $\left\{K_{i}\right\}$ and a quantum channel $\Psi: \rho \mapsto U_{\epsilon} \Phi(\rho) U_{\epsilon}^{\dagger}$, where $U_{\epsilon}=\exp (-i \epsilon H) \in \mathcal{U}(\mathcal{X})$. We get:

$$
G_{\mathrm{ch}}(\Phi, \Psi ; \sigma)=1+\sum_{i j}\left|\operatorname{Tr} \sigma K_{i}^{\dagger} U_{\epsilon} K_{j}\right|^{2}-\sum_{i j}\left|\operatorname{Tr} \sigma K_{i}^{\dagger} K_{j}\right|^{2} .
$$


Now, we concentrate on the change of the quantum channel superfidelity under the change of $\epsilon$. As we are interested only in small values of $\epsilon$, we expand Eq. (40) up to the linear term in the Taylor series. For small values of $\epsilon$, we get:

$$
G_{\mathrm{ch}} \approx 1-2 \epsilon \sum_{i j} \Im \operatorname{Tr} \sigma K_{i}^{\dagger} H K_{j} \overline{\operatorname{Tr} \sigma K_{i}^{\dagger} K_{j}}
$$

Note that this depends on the value of the observable $H$ of the operator $K_{j} \sigma K_{i}^{\dagger}$.

\section{Sensitivity to Hamiltonian parameters}

In this section, we will show how the channel superfidelity is affected by errors in the system Hamiltonian parameters. First, we will show analytical results for a single qubit system at a finite temperature. Next, we show numerical results for a simple, three-qubit spin chain.

\subsection{Single qubit at a finite temperature}

A single qubit at a finite temperature is described by the master equation

$$
\begin{aligned}
\dot{\rho}(t)= & -\mathrm{i} \frac{\Omega+\epsilon}{2}\left[\sigma_{z}, \rho(t)\right]+\gamma_{+}\left(\sigma_{-} \rho(t) \sigma_{+}-\frac{1}{2}\left\{\sigma_{+} \sigma_{-}, \rho(t)\right\}\right) \\
& +\gamma_{-}\left(\sigma_{+} \rho(t) \sigma_{-}-\frac{1}{2}\left\{\sigma_{-} \sigma_{+}, \rho(t)\right\}\right),
\end{aligned}
$$

where $\sigma_{+}=|1\rangle\langle 0|, \sigma_{-}=\sigma_{+}^{\dagger}$ and $\epsilon$ is the error in $\Omega$. Our goal is to calculate the quantum channel superfidelity between the case when there is no error in $\Omega$, i.e. $\epsilon=0$ and the case with error in $\Omega$. Henceforth, we will assume $\gamma_{-}=\gamma_{+}=1$ for clarity.

For a given time $T$, Eq. (42) may be rewritten as

$$
\rho(T)=\Phi_{T}^{\epsilon}(\rho(0))
$$

where $\Phi_{T}^{\epsilon}$ is a quantum channel in the quantum dynamical semigroup. A natural representation $M_{\Phi_{T}^{\epsilon}}$ for the channel $\Phi_{T}^{\epsilon}$ may be found as [23]:

$$
M_{\Phi_{T}^{\epsilon}}=\mathrm{e}^{-F T}
$$

where

$$
\begin{aligned}
F= & -\mathrm{i} \frac{\Omega+\epsilon}{2}\left(\mathbb{1} \otimes \sigma_{z}-\sigma_{z} \otimes \mathbb{1}\right)-\sigma_{-} \otimes \sigma_{-}-\sigma_{+} \otimes \sigma_{+}+ \\
& +\frac{1}{2}\left(\sigma_{+} \sigma_{-} \otimes \mathbb{1}+\sigma_{-} \sigma_{+} \otimes \mathbb{1}+\mathbb{1} \otimes \sigma_{+} \sigma_{-}+\mathbb{1} \otimes \sigma_{-} \sigma_{+}\right) .
\end{aligned}
$$


In this representation, we may rewrite Eq. (43) as

$$
\operatorname{res}(\rho(T))=M_{\Phi_{T}^{\epsilon}} \operatorname{res}(\rho(0))
$$

The Choi-Jamiołkowski representation of the channel $\Phi_{T}$ is given by $D_{\Phi_{T}^{\epsilon}}=$ $\left(M_{\Phi_{T}}^{\epsilon}\right)^{\mathrm{R}}$. Here, $M^{\mathrm{R}}$ denotes the reshuffle operation on matrix $M$ [14]. Now, it is simple to find the Kraus form of the channel $\Phi_{T}^{\epsilon}$. The Kraus operators are related to the eigenvalues $\lambda_{i}$ and eigenvectors $\left|\lambda_{i}\right\rangle$ of $D_{\Phi_{T}^{\epsilon}}$ in the following manner:

$$
K_{i}^{\Phi_{T}^{\epsilon}}=\sqrt{\lambda_{i}} \text { unres }\left(\left|\lambda_{i}\right\rangle\right)
$$

Inserting these Kraus operators into Eq. (28), we get

$$
G_{\mathrm{ch}}\left(\Phi_{T}^{0}, \Phi_{T}^{\epsilon} ; \rho\right)=1-2 \mathrm{e}^{-2 T}(1-\cos \epsilon T) \rho_{00}(0) \rho_{11}(0),
$$

where $\rho_{i i}(0)=\langle i|\rho(0)| i\rangle$. Note that we get $G_{\mathrm{ch}}=1$ in two cases. First, for large $T$ and second when $\epsilon T=\frac{\pi}{2}$. As we are mainly interested in small values of $\epsilon$, we expand $\cos \epsilon T$ up to the second term in the Taylor series. We get:

$$
G_{\mathrm{ch}}\left(\Phi_{T}^{0}, \Phi_{T}^{\epsilon} ; \rho\right) \approx 1-\epsilon^{2} T^{2} \mathrm{e}^{-2 T} \rho_{00}(0) \rho_{11}(0)
$$

In this setup, the channel superfidelity has a quadratic dependence on the error parameter $\epsilon$. This should be compared with the results in Sect. 4.2.

\subsection{Quantum control example}

In this section, we consider a three-qubit spin chain with dephasing interactions with the environment. We will consider piecewise constant control pulses. The time evolution of the system is governed by the equation:

$$
\dot{\rho}(t)=-\mathrm{i}[H, \rho(t)]+\gamma\left(\sigma_{z} \rho(t) \sigma_{z}-\rho(t)\right),
$$

where $H=H_{\mathrm{d}}+H_{\mathrm{c}}$. Here $H_{\mathrm{d}}$ is the drift term of the Hamiltonian given by

$$
H_{\mathrm{d}}=J \sum_{i=1}^{2} \sum_{\alpha \in\{x, y, z\}} \sigma_{\alpha}^{i} \sigma_{\alpha}^{i+1}
$$

where $\sigma_{\alpha}^{i}$ denotes $\sigma_{\alpha}$ acting on site $i$. We set the control Hamiltonian $H_{\mathrm{c}}$ to:

$$
H_{\mathrm{c}}=\sum_{i}^{N} h_{x}\left(t_{i}\right) \sigma_{x}^{1}+h_{y}\left(t_{i}\right) \sigma_{y}^{1},
$$


Fig. 2 Quantum channel superfidelity as a function of noise in the system's control pulses. The shaded area represents the range of the achieved channel superfidelity, and the black line is the average channel superfidelity
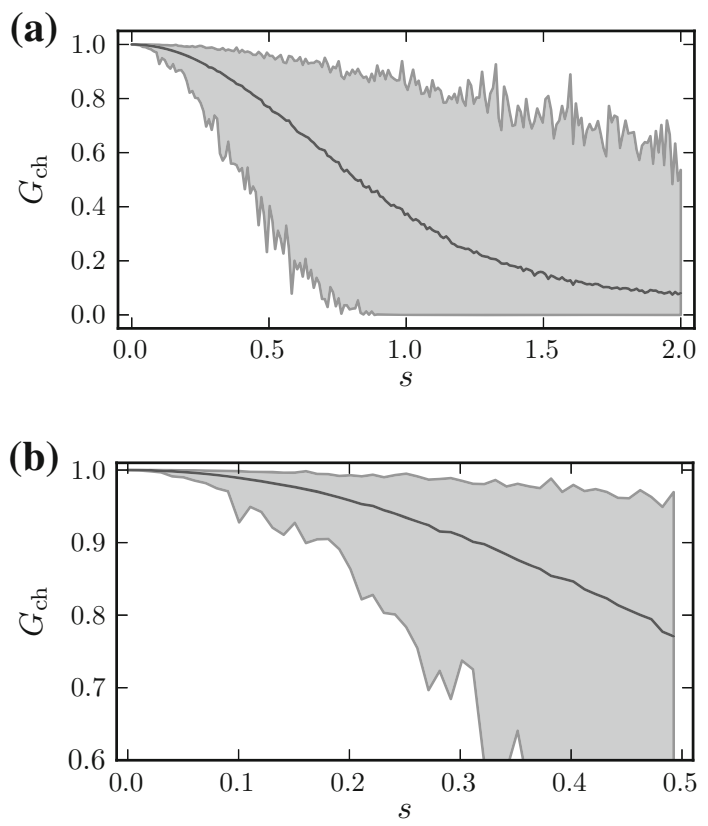

where $h_{x}\left(t_{i}\right)$ and $h_{y}\left(t_{i}\right)$ denote the control pulses in the time interval $t_{i}$. We set the target to be

$$
U_{\mathrm{T}}=\mathbb{1} \otimes 1 \otimes \sigma_{x},
$$

i.e. a NOT gate on the third qubit. We fixed the number of time intervals $N=64$, the total evolution time $T=6.1$ and the maximum amplitude of a single control pulse $\forall k \in x, y \max \left(\left|h_{k}\right|\right)=10$.

First, we optimize control pulses for the system, such that we achieve a high fidelity of the gate $U_{T}$. Next, to each control pulse we add a noise term $h_{\epsilon}$ witch has a normal distribution, $h_{\epsilon}=N(0, s)$. Figure 2 shows the change of $G_{\mathrm{ch}}$ as a function of the standard deviation $s$. We have conducted 100 simulations for each value of $s$. As expected, the quantum channel superfidelity decreases slowly for low values of $s$. After a certain value, the decrease becomes rapid. As values of $s$ increase, the minimum and maximum achieved fidelity diverge rapidly. This is represented by the shaded area in Fig. 2. We can approximate the average value of the channel fidelity as $\left\langle G_{\mathrm{ch}}\right\rangle \approx 1-c s^{2}$. Fitting this function to the curve shown in Fig. $2 \mathrm{~b}$ gives a relative error which is less then $0.5 \%$.

\section{Conclusions}

We have studied the superfidelity of a quantum channel. This quantity allows us to provide an upper bound on the fidelity of the output of two quantum channels. We shown an example of application of this quantity to a unitary and an erasure 
channel. The obtained superfidelity can be easily limited from above by the product w eigenvalues of the input state $\sigma$ and the result of the erasure channel $\xi$.

Furthermore, as shown in our examples, the quantum channel superfidelity may have potential applications in quantum control theory as an easy to compute figure of merit of quantum operations. In a simple setup, where the desired quantum channel is changed by a unitary transformation $U_{\epsilon}=\exp (-\mathrm{i} \epsilon H)$ we get a linear of the decrease of channel superfidelity on the noise parameter $\epsilon$. On the other hand, when we introduce the noise as a control error in a single qubit quantum control setup, we get a quadratic dependence on the noise parameter.

Finally, we shown numerical results for a more complicated system. We calculated the quantum channel superfidelity for a three-qubit quantum control setup. First, we found control pulses which achieve a high fidelity of the desired quantum operation, next we introduced Gaussian noise in the control pulses. Our results show that the quantum channel superfidelity stayed high for a wide range of the noise strength.

Acknowledgments We would like to thank Piotr Gawron for inspiring discussions. ŁP was supported by the Polish National Science Centre under decision number DEC-2012/05/N/ST7/01105. ZP supported by the Polish National Science Centre under the post-doc programme, decision number DEC2012/04/S/ST6/00400.

Open Access This article is distributed under the terms of the Creative Commons Attribution 4.0 International License (http://creativecommons.org/licenses/by/4.0/), which permits unrestricted use, distribution, and reproduction in any medium, provided you give appropriate credit to the original author(s) and the source, provide a link to the Creative Commons license, and indicate if changes were made.

\section{References}

1. Viola, L., Knill, E., Lloyd, S.: Dynamical decoupling of open quantum systems. Phys. Rev. Lett. 82(12), 2417 (1999)

2. Viola, L., Lloyd, S., Knill, E.: Universal control of decoupled quantum systems. Phys. Rev. Lett. 83(23), 4888 (1999)

3. Viola, L., Knill, E.: Robust dynamical decoupling of quantum systems with bounded controls. Phys. Rev. Lett. 90(3), 037901 (2003)

4. Dahleh, M., Peirce, A., Rabitz, H.: Optimal control of uncertain quantum systems. Phys. Rev. A 42(3), 1065 (1990)

5. Dong, D., Petersen, I.R.: Sliding mode control of quantum systems. New J. Phys. 11(10), 105033 (2009)

6. James, M.: Risk-sensitive optimal control of quantum systems. Phys. Rev. A 69(3), 032108 (2004)

7. D'Helon, C., Doherty, A., James, M., Wilson, S.: Quantum risk-sensitive control. In : 45th IEEE Conference on Decision and Control, 2006, pp. 3132-3137. IEEE (2006)

8. Pawela, Ł., Sadowski, P.: Various Methods of Optimizing Control Pulses for Quantum Systems with Decoherence. arXiv preprint arXiv:1310.2109, (2013)

9. Pawela, Ł., Puchała, Z.: Quantum control robust with respect to coupling with an external environment. Quantum Inf. Process. 14(2), 437-446 (2015)

10. Gawron, P., Kurzyk, D., Pawela, Ł.: Decoherence effects in the quantum qubit flip game using markovian approximation. Quantum Inf. Process. 13, 665-682 (2014)

11. Pawela, Ł., Puchała, Z.: Quantum control with spectral constraints. Quantum Inf. Process. 13, 227-237 (2014)

12. Raginsky, M.: A fidelity measure for quantum channels. Phys. Lett. A 290(1), 11-18 (2001)

13. Piani, M., Watrous, J.: All entangled states are useful for channel discrimination. Phys. Rev. Lett. 102(25), 250501 (2009) 
14. Bengtsson, I., Życzkowski, K.: Geometry of Quantum States: An Introduction to Quantum Entanglement. Cambridge University Press, Cambridge, UK (2006)

15. Nielsen, M.A., Chuang, I.L.: Quantum Computation and Quantum Information. Cambridge University Press, Cambridge (2000)

16. Puchała, Z., Miszczak, J.A., Gawron, P., Gardas, B.: Experimentally feasible measures of distance between quantum operations. Quantum Inf. Process. 10(1), 1-12 (2011)

17. Miszczak, J.A., Puchała, Z., Horodecki, P., Uhlmann, A., Życzkowski, K.: Sub- and super-fidelity as bounds for quantum fidelity. Quantum Inf. Comput. 9(1), 103-130 (2009)

18. Mendonça, P.E., Napolitano, RdJ, Marchiolli, M.A., Foster, C.J., Liang, Y.-C.: Alternative fidelity measure between quantum states. Phys. Rev. A 78(5), 052330 (2008)

19. Puchała, Z., Miszczak, J.A.: Bound on trace distance based on superfidelity. Phys. Rev. A 79(2), 024302 (2009)

20. Watrous, J.: Theory of Quantum Information. https://cs.uwaterloo.ca/ watrous/CS766/LectureNotes/ all.pdf (2011)

21. Ekert, A.K., Alves, C.M., Oi, D.K.L., Horodecki, M., Horodecki, P., Kwek, L.C.: Direct estimations of linear and nonlinear functionals of a quantum state. Phys. Rev. Lett. 88(21), 217901 (2002)

22. Horn, R., Johnson, C.: Topics in Matrix Analysis. Cambridge University Presss, Cambridge (1991)

23. Havel, T.F.: Robust procedures for converting among Lindblad, Kraus and Matrix representations of quantum dynamical semigroups. J. Math. Phys. 44(2), 534-557 (2003) 